\title{
Obstetrics state in non-epizootic area during early epidemic of COVID-19
}

\author{
Chao Ji ${ }^{1}$, Jiongbo Wang ${ }^{1}$, Yichen Qin², Xipeng Zhang ${ }^{1}$, and Qian Zhang ${ }^{1}$ \\ ${ }^{1}$ Qingdao Municipal Hospital Group \\ ${ }^{2}$ The Third People's Hospital of Qingdao
}

August 3, 2020

\begin{abstract}
Abstract Objective To study the impacts of obstetric complications and anxiety among pregnant women in non-epizootic area which are caused by COVID-19. Design Retrospective cohort. Setting China. Population or sample the women who delivered in the third trimester of pregnancy in Qingdao Municipal hospital for the year the months March and April, 2019 and 2020. Method This paper is based on the clinical data from This paper makes a detailed analysis of the problems that can arise because of special circumstances related with delivery history, personal physical symptoms, anxiety index, adverse complications as well as neonatal birth weight. Result Comparing with the same period between 2019 and 2020 , maternal age $(32.12 \pm 5.32 \mathrm{vs} 31.01 \pm 4.21, \mathrm{P}<0.05)$ and the ratio of macrosomia $(12.10 \% \mathrm{vs} 2.53 \%)$ are increasing steadily. Meanwhile, times of prenatal visits $(10.49 \pm 2.15 \mathrm{vs} 10.89 \pm 1.81, \mathrm{P}<0.05)$ and score of $\mathrm{PHQ}-9(1.46+-2.09 \mathrm{vs} 4.96+-1.38, \mathrm{P}<0.05)$ are to fall significantly 。 Conclusion It is helpful to relieve the negative emotion of fear and anxiousness for the pregnant women if they can be encouraged and soothed by social support. The leading cause why regular checkups are decreasing is due to the fear of the epidemic and the transport restrictions, which, in return, may result in a lack of effective management in fetal weight. The COVID-19 had no giant effect on the third trimester of pregnant women. Government as well as obstetric practitioners should strengthen the education of fetal weight management during the pregnancy. Keywords Pregnancy; COVID-19; obstetric complications; anxiety Tweetable abstract The COVID-19 had no giant effect on the third trimester of pregnant women in the non-epizootic area China.
\end{abstract}

Obstetrics state in non-epizootic area during early epidemic of COVID-19

Chao $\mathrm{Ji}^{\mathrm{a}}$, Jiongbo Wang ${ }^{\mathrm{a}}$, Yichen Qin ${ }^{\mathrm{b}}$, Xipeng Zhang ${ }^{\mathrm{a}}$, Qian Zhang ${ }^{\mathrm{a} *}$

a. Qingdao Municipal Hospital, 266011, Qingdao, China

b. The Third People's Hospital of Qingdao, 266041, Qingdao, China

Corresponding author

Name: Qian Zhang

Address: Qingdao Municipal Hospital, 266011, Qingdao, China

Telephone number: +86-18561728229

Email:zhangqian6368@sina.com

\section{Running title}

Obstetrics state during early epidemic of COVID-19

\section{Abstract}




\section{Objective}

To study the impacts of obstetric complications and anxiety among pregnant women in non-epizootic area which are caused by COVID-19.

\section{Design}

Retrospective cohort.

\section{Setting}

China.

\section{Population or sample}

the women who delivered in the third trimester of pregnancy in Qingdao Municipal hospital for the year the months March and April, 2019 and 2020.

\section{Method}

This paper is based on the clinical data from This paper makes a detailed analysis of the problems that can arise because of special circumstances related with delivery history, personal physical symptoms, anxiety index, adverse complications as well as neonatal birth weight.

\section{Result}

Comparing with the same period between 2019 and 2020, maternal age $(32.12 \pm 5.32 \mathrm{vs} 31.01 \pm 4.21, \mathrm{P}<0.05)$ and the ratio of macrosomia(12.10\%vs2.53\%)are increasing steadily. Meanwhile, times of prenatal visits $(10.49 \pm 2.15 v s 10.89 \pm 1.81, \mathrm{P}<0.05)$ and score of $\mathrm{PHQ}-9(1.46+-2.09 \mathrm{vs} 4.96+-1.38, \mathrm{P}<0.05)$ are to fall significantly。

\section{Conclusion}

It is helpful to relieve the negative emotion of fear and anxiousness for the pregnant women if they can be encouraged and soothed by social support. The leading cause why regular checkups are decreasing is due to the fear of the epidemic and the transport restrictions, which, in return, may result in a lack of effective management in fetal weight. The COVID-19 had no giant effect on the third trimester of pregnant women. Government as well as obstetric practitioners should strengthen the education of fetal weight management during the pregnancy.

\section{Keywords}

Pregnancy; COVID-19; obstetric complications; anxiety

\section{Tweetable abstract}

The COVID-19 had no giant effect on the third trimester of pregnant women in the non-epizootic area China.

\section{Funding}

No funding.

\section{Introduction}

A deadly virus called coronavirus disease 2019(COVID-19)is running rampant across the globe. The first cases of COVID-19 were reported from Wuhan, China in Jan,2020 and spread across continents with an increasing number of cases and deaths day by day. On January 30, 2020, the World Health Organization (WHO) labeled the outbreak as a Public Health Emergency of International Concern (PHEIC). On February 12, 2020, the WHO named the disease, caused by this coronavirus, "Coronavirus Disease 2019 (COVID19)". More than 12million people worldwide have been infected with COVID-19 and 500 thousands people have died because of an infection with COVID-19. The WHO asserted that along the most common way of infection by respiratory droplets, is a transmission by aerosol, person-to-person and surface possible. 
Although it failed to prove that pregnant women, as a vulnerable group of people, suffer from high risk of vertical transmission of COVID-19 in the late third trimester reported ${ }^{[1]}$, they still be frightened and need additional precautions. Not only the immunosuppression of pregnancy, such as gestational diabetes mellitus (GDM) and preeclampsia, may impact severity of symptoms caused by COVID-19, also the negative emotions like anxiety, depression and stress can have a negative effect. The social instability also has negative influence on pregnant women which may lead to a passive perinatal outcome. Based on current researches, more attention was paid to the COVID-19 infected pregnant women. Few obstetric original researches existed about adverse impact on the health of pregnant women in the non-epizootic area.

Given the importance of the issue and the lack of sufficient evidence, the present study aimed to review obstetric data of our hospital to find out the influence of this disease on the Chinese pregnant women.

\section{Materials and methods}

We conducted a retrospective by reviewing 822 medical records of delivered women whom admitted to Qingdao Municipal Hospital from March 1 to April 30, 2019 and 2020.Information we collected can be summarized as follows. The maternal characteristics which include delivery history, personal physical signs, adverse complications as well as neonatal birth weight were analyzed. The patient health questionnaire(PHQ9 ) is the tool for anxiety evaluation. All information was obtained from a customized data collection form. We compared the data of two years and found evidence to prove the detriment due to COVID-19.All descriptive and statistical analyses were performed using SPSS version 26.0. A p value of less than 0.05 was considered to indicate statistical significance (two-sided).

\section{Result}

A total 822 pregnant women were involved into our research and 347 delivered in 2020 and the rest in 2019. The table 1 lists the comparison of two groups about mother characteristics. Maternal age $(32.12 \pm 5.32 \mathrm{vs} 31.01 \pm 4.21)$ and times of prenatal visits $(10.49 \pm 2.15 \mathrm{vs} 10.89 \pm 1.81)$ had the remarkable statistical significance $(\mathrm{P}<0.05)$. Score of PHQ-9 in 2019(4.96 \pm 1.38$)$ was higher than it in $2020(1.46 \pm 2.09)(\mathrm{P}<0.05)$. There was no conspicuous distinction in Gestational weight gain (GWG).

On the concept of adverse complication, macrosomia $(\mathrm{n}=12)$ was the unique meaningfully contrastive index in the Table 2. The other complications failed to perform difference in this two years, as well number of caesarean.

There were 486 newborn in 2019 and 355 in 2020 according to table 3 . The birth weight of two group (3440.96 \pm 540.22 vs3371.55 \pm 526.24$)$ exhibited the consistency with $\mathrm{P}>0.05$.

\section{Discussion}

The WHO constantly publishes the latest worldwide timeline of the evolution of the COVID-19.It became a global pandemic due to the characteristics of high infectivity, transmitting speed and fatality ${ }^{[2]}$. It has spread to over 200 countries and claimed 50thousands people's lives, since it emerged in 2020.

With the development of modern transport, tens of millions of people have been leaving their homelands in search of a better job or education. But come the spring festival, not only migrant workers, also students rush back to their hometown. Due to the increased number of people travelling during Spring festival, the COVID-19 can spread easier and faster. Governments take varying degrees of preventive measures, including asking for mask wearing, shutting down the businesses, schools and patients quarantine, etc. At 
present, global surveillance and control faced with serious challenge, the prevention and control of COVID19 infection among pregnant women and the potential hazard of vertical transmission have come to the concerned schedule. However, the original research about adverse impact on the health pregnant women in the non-epizootic area was rarely.

There are three transmission elements of infectious diseases : source of infection, route of transmission and susceptible population. The maternal immune system of pregnant women is in a dilemma that it need to establish immunologic tolerance to the allogeneic fetus, while to defend the bodies from the invasion of pathogenic microorganisms. The maternal immunological states keep being in flux through the pregnant progress. Pregnancy drives women into a partial immune deficiency state ${ }^{[3]}$. It takes for granted that pregnant women own more possibility of infection on account of the COVID-19. Whereas some latest Chinese researches ${ }^{[1,4,5]}$ drew the conclusion that pregnant women presented the equal opportunity of COVID-19 infection, compared with non-pregnant patients. This consequence shocks the common sense of the public, but still it may come to light if more cases are analyzed and geographic limitation is ruled out. Several evidences could prove the infection insurability between mothers and fetuses. We must keep vigilant to its potential risks. Researches prove that infection could activate the immune system and rise the level of some immune factors such as TNF- $\alpha$ and IL-17a, which could lead to brain development abnormalities, preterm labor, even fetal loss ${ }^{[6,7]}$. And fever as the most common and initial symptom of COVID-19 infection could be associated with increased attention-deficit/hyperactivity disorder in the offspring ${ }^{[3]}$.

It was obviously that the average maternal age of the pregnant in 2020 is more advanced compare with 2019 ,even merely about one year gap. This result confirmed the global trend of postponement of child birth, both in developing and developed countries. An increasing number of well-educated women whom stepping into the workforce, with better and higher education, work and socioeconomic pressure, attitude to marriage and fertility make their maternal age been prolonged continually. The average age of primipara was 21 years in 1972 and in 2016 it reached 26.3 years ${ }^{[8]}$.Advanced maternal age is usually defined as being 35years, which is believed to relate to varying complication and adverse outcomes ${ }^{[9]}$.In our study, maternal age of two group women was less than 35 and little different existed in absolute value. Therefore, the impact of age can be ignored from the discussion of the result.

We can precisely find out the recession of quantity of deliveries and frequency of prenatal visits in 2020 in our research, compared to 2019. Prenatal care services aim at lowering maternal and neonatal morbidity and mortality and improving the quality of global population. By means of prenatal physical examination, history taking and imagological and laboratory examination, obstetricscan offer the helpful advice and suggestion for lifestyles, identify the complications associated with pregnancy and provide the appropriate treatment, the treatment that pregnant women might benefit from. For example, the pregnant women whose frequency of prenatal visits was insufficient suffered higher risk of perinatal complications, especially macrosomia ${ }^{[10]}$, which is the same result in our research. And the prenatal care can efficiently stem the congenital syphilis cases $^{[11]}$ and pregnancy-induced hypertension ${ }^{[12]}$. We believe that people, especially the pregnant, reduce the need of regular hospital check-ups, even refuse to walk out the door to preventCOVID-19 because of fear and worry. It also means they chose to cut down the frequency of prenatal care services as well as fail to get the professional obstetric advises from doctors, which might avoid certain obstetric complications. What's more, it is well-known that the childbearing age women held a large percentage of floating population and migrant workers. The non-indigenous pregnant women hold nearly half of the patients of our clinic. Atthe same time, the Chinese government took a series of measurements to cut-off the dissemination way. Because of self-protection of native pregnant and traffic restriction of ecdemic ones, the selective prenatal visits and patient's loss explained the change of frequency of prenatal visits.

In China, $11.1 \%$ and $10.3 \%$ of pregnant women were suffering from anxiety and depression, respectively ${ }^{[13]}$.On the surface, pregnancy is a constantly changing process for physiological structure. In fact, endogenous hormone levels and stimulation from external environment catalyze psychological activities. It is a susceptible period that the negative life stuff could lead to prenatal anxiety and depression, which have been global public health problems in the last decade ${ }^{[14]}$.Except for pregnancy-related anxiety and depression, the panic for 
COVID-19, as an updated socially channel, aggravates these pathogenic factors. During this epidemic, the worldwide economy suffered a hard blow. Pregnant women, especially elder ones, confront salary cuts and unemployment, which threatens their socioeconomic status, which accelerates depression and age-related anxiety ${ }^{[13]}$. While prenatal anxiety and depression to a certain extent may be treated as a common occurrence during pregnancy, an important life-phase for a women, from a girl to a mom, high levels of them may have long-lasting adversely influence on both the her behavior and her child's future development, such as ${\text { preterm } \text { birth }^{[15]} \text {, low birth weight }}^{[16]}$ and weight increase ${ }^{[17]}$. Nonetheless, our research failed to validate the hypothesis above and a opposite result showed. The results of psychological assessment during hospital admission show equivalent score of two years' women. Women's weight increase, rate of preterm birth and neonates' birth weight in our research suggest the parallel consequence. It turned out that pregnant women, who delivered in March and April, 2020 avoid the additional anxiety and depression on account of COVID-19. The feasible explanation is the better family concern. When it comes to scores of health related quality of life (HRQoL), pregnant women, especially during the third trimester, got the significantly lower one than nonpregnant women of the same age, and some sociodemographic factors which could improve the well-being, involving the social support and partner support. Researches declared the importance of social support during pregnancy, especially family care ${ }^{[16]}$. Pregnant women who are in absence of social support tend to be pessimistic and suffer from low self-esteem or self-worth ${ }^{[17]}$. Thanks to the epidemic, less work makes family members pay more attention to the pregnant ones, which can moderate the stimulation from life stuff and exoteric environment. When a pregnant woman gets adequate social support from her family and friends who provide nutrient-rich diet, physical and mental solicitude, and medical suggestion, she is motivated to take care of herself which is a true indicator of her HRQoL, which could decrease negative affection such as depression and anxiety. Moreover, more consideration from the partner could elevate marital satisfaction and the pregnant women who have higher marital satisfaction have a healthier life attitude, which means satisfying diets and less abnormal psychological problems ${ }^{[18]}$. A long-term and stable marriage relationship could increase the happiness and counteract external pressure from the society.

Meeting the optimal fetal development and sufficient nutrition for mother is the basic requirement for a healthy, balanced diet. A study with Asian subjects indicated that the total energy expenditure of women during their pregnancy was significantly lower than that before they became pregnant ${ }^{[19]}$.In the Chinese tradition, a big newborn symbolizes jubilation and abundance. Although many women realize the importance of balanced diet during pregnancy, lack of professional introduction and food cravings, a strong impetus ${ }^{[20]}$, during pregnancy facilitate excess calorie intake. As a result, women get more GWG during this period. In current, thanks to the propaganda and education from obstetric stuff, Chinese pregnant women have become sensitive and learned to control the GWG, based on appropriate growth rate of the fetus. This achievement can be found out in our research that there was no significant disparity of GWG in 2019 and 2020. However, clinically, less GWG might correspond to heavier fetal weight. In our study, the average weight of newborns in 2020 was equal to it in 2019, whereas the rate of macrosomia was higher. Physical activity is the main pathway to accelerate the energy expenditure. The benefits of physical activity during pregnancy include improved physical fitness, reduced risk of excessive weight gain, reduced risk of preeclampsia and pre-term birth, reduced low back pain, improved sleep, reduced anxiety and depressive symptoms, and improved health perception and self-reported body image ${ }^{[21]}$.It is recommended that, without medical restrictions of physical activity and under the applicable exercise intensity, pregnant women should aim to perform $30 \mathrm{~min}$ for most days of the week with the guidelines published by the United States or 60 min of aerobic exercise for $2-3$ times per week suggested by the Japanese guidelines ${ }^{[22]}$. Nevertheless, the pregnant women who reach the recommended level of physical activity are in the minority both in the developed and developing countries ${ }^{[23]}$. On one hand, the Asia's idea about lifestyle during duration of pregnancy is highly diverse compared to western world, especially in China. The continuation of the family line is an extremely important affair in Chinese tradition. Hence pregnant women spontaneously avoid doing some things which might have negative influence on pregnancy and fetus and the intensity of exercise is lower than the occidental. On another hand, A meta-analysis found out an opposite correlation between physical activity and the occurrence of macrosomia ${ }^{[24]}$. This is a possible explanation for high level of macrosomia emerging in our study. 


\section{Limitation}

Our study has the following limitations: First, inconsideration of Chinese government's effective control and the limited sample resource, the study we conducted is only in one regional hospital in Qingdao and the cases were from the same month of two different years. The quantities and representativeness of sample was finite. Second, as a cross-sectional design study, we could not identify the ultimate influence on the pregnant who was in the first two trimesters. Third; the hospital admission psychological assessment is conducted by PHQ-9 rather than psychologist, which may weaken the accuracy. Thus, the conclusion can be only for reference for analyzing the global obstetric situation under the shadow of COVID- 19. Further information could be collected to verify the validity of our research.

\section{Conclusion}

COVID-19 is a high-scale catastrophes for mankind, not only the severe symptoms and death rate also may affect pregnant woman in several aspects. It doesn't only reduce the regular prenatal examination also reduce the basic exercise during all terms of pregnancy. The government and medical practitioners should take more measures to enhance the antenatal care service both online and off and eliminate the indefinite information. Efficient epidemic control is the fundamental solution to exclude the negative influence on the pregnant due to COVID-19.

\section{Disclosure of interests}

None declared. Completed disclosure of interest forms are available to view online as supporting information.

\section{Contribution to authorship}

Conceptualisation was by Chao Ji and Qian Zhang; data curation was by Jiongbo Wang, Xipeng Zhang and Xipeng Zhang; formal analysis was by Chao Ji and Jiongbo Wang; and writing was by Chao Ji, Yichen Qin, Qian Zhang..

\section{Details of ethics approval}

The current study was reviewed and qualified as an exemption by the Institutional Review Board of Qingdao Municipal Hospital, Qingdao, China (2020临审字第052号) on 29 July 2020. Because the study analyzed publicly available data, no consent was obtained from the women by the authors. The principles expressed in the Declaration of Helsinki have been adhered to.

\section{Funding}

No funding.

\section{Acknowledgements}

None.

\section{References}

1. H, C., et al., Clinical characteristics and intrauterine vertical transmission potential of COVID-19 infection in nine pregnant women: a retrospective review of medical records. Lancet (London, England), 2020. 395(10226): p. 809-815.

2. Singhal, T., A Review of Coronavirus Disease-2019 (COVID-19). Indian J Pediatr, 2020. 87(4): p. 281-286. 
3. H, L., et al., Why are pregnant women susceptible to COVID-19? An immunological viewpoint. Journal of reproductive immunology, 2020. 139: p. 103122.

4. H, Z., et al., Clinical analysis of 10 neonates born to mothers with 2019-nCoV pneumonia. Translational pediatrics, 2020. 9(1): p. 51-60.

5. Wang, X., et al., A case of 2019 Novel Coronavirus in a pregnant woman with preterm delivery. Clin Infect Dis, 2020.

6. GB, C., et al., The maternal interleukin-17a pathway in mice promotes autism-like phenotypes in offspring. Science (New York, N.Y.), 2016. 351(6276): p. 933-9.

7. M, F., et al., Systemic Inflammatory Response to Malaria During Pregnancy Is Associated With Pregnancy Loss and Preterm Delivery. Clinical infectious diseases : an official publication of the Infectious Diseases Society of America, 2017. 65(10): p. 1729-1735.

8. Quoctrung B, Miller CC. The Age That Women Have Babies: How a Gap DividesAmerica. Available athttps://www.nytimes.com/interactive/2018/08/04/upshot/up-birth-agegap.html?searchResultPosition=, Accessed October 10, 2019. 1.

9. MA, M., et al., Advanced maternal age pregnancy and its adverse obstetrical and perinatal outcomes in Ayder comprehensive specialized hospital, Northern Ethiopia, 2017: a comparative cross-sectional study. BMC pregnancy and childbirth, 2020. 20(1): p. 60.

10. N, B.Y., et al., Inadequate Prenatal Visit and Home Delivery as Determinants of Perinatal Outcomes: Does Parity Matter? Journal of pregnancy, 2019. 2019: p. 9024258.

11. EZ, M., et al., Attributable fraction of congenital syphilis due to the lack of prenatal care. Revista da Sociedade Brasileira de Medicina Tropical, 2019. 52: p. e20180532.

12. S, O., et al., Correction: Associations of psychosocial factors with pregnancy healthy life styles. PloS one, 2018. 13(5): p. e0197389.

13. X, M., et al., The impact of resilience on prenatal anxiety and depression among pregnant women in Shanghai. Journal of affective disorders, 2019. 250: p. 57-64.

14. A, B., et al., Identifying the women at risk of antenatal anxiety and depression: A systematic review. Journal of affective disorders, 2016. 191: p. 62-77.

15. ZB, K. and B. M, The association between pregnancy-specific anxiety and preterm birth: a cohort study. African health sciences, 2018. 18(3): p. 569-575.

16. YT, K., et al., Prevalence and Risk Factors of Maternal Anxiety in Late Pregnancy in China. International journal of environmental research and public health, 2016. 13(5).

17. A, K., et al., Perceived psychosocial stress and gestational weight gain among women with gestational diabetes. PloS one, 2017. 12(3): p. e0174290.

18. S, O., et al., Associations of psychosocial factors with pregnancy healthy life styles. PloS one, 2018. 13(1): p. e0191723.

19. S, N., et al., Current Insights into Exercise-based Cardiac Rehabilitation in Patients with Coronary Heart Disease and Chronic Heart Failure. International journal of sports medicine, 2020.

20. LE, F., et al., Dietary Change during Pregnancy and Women's Reasons for Change. Nutrients, 2018. $10(8)$.

21. AL, H., et al., Attitudes, barriers and enablers to physical activity in pregnant women: a systematic review. Journal of physiotherapy, 2018. 64(1): p. 24-32. 
22. CWH, C., A.Y. E, and L. BMH, Effectiveness of Physical Activity Interventions on Pregnancy-Related Outcomes among Pregnant Women: A Systematic Review. International journal of environmental research and public health, 2019. 16(10).

23. A, L., et al., Relationship between Physical Activity and Health Related Quality of Life among Pregnant Women. African journal of reproductive health, 2018. 22(3): p. 80-89.

24. M, M., et al., The impact of physical activity during pregnancy on maternal weight and obstetric outcomes. Ginekologia polska, 2018. 89(2): p. 80-88.

Supporting information

Table 1 Maternal characteristics

$$
2020(n=347) 2019(n=475) \quad \mathrm{P}
$$

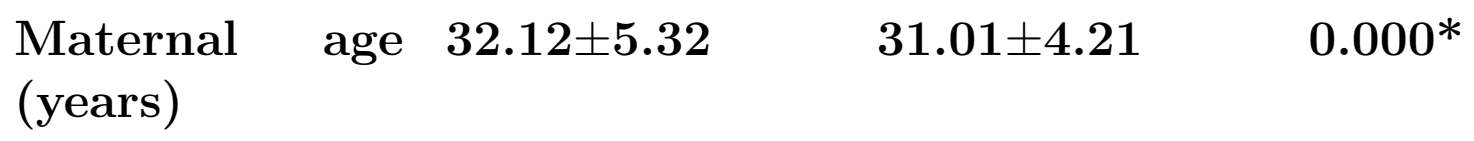

$\begin{array}{llll}\text { Delivery } & 39.18 \pm 2.21 & 39.35 \pm 1.57 & 0.202 \\ \text { gestational } & & & \\ \text { week(weeks) } & & & \end{array}$

$\begin{array}{llll}\text { Times of preg- } & 2.14 \pm 1.19 & 2.07 \pm 1.15 & 0.411 \\ \text { nancy } & \end{array}$

Times of deliv- $1.47 \pm 0.55 \quad 1.48 \pm 0.58 \quad 0.937$ ery 


$$
2020(n=347) 2019(n=475) \quad \mathrm{P}
$$

$\begin{array}{lllll}\text { Body } & \text { mass } & 28.40 \pm 3.49 & 28.23 \pm 3.67 & 0.513\end{array}$ $\operatorname{index}\left(\mathrm{kg} / \mathrm{m}^{2}\right)$

Times of prena- $10.49 \pm 2.15 \quad 10.89 \pm 1.81 \quad 0.004^{*}$ tal visits
GWG(kg)
$14.99 \pm 4.99$
$15.61 \pm 5.32$
0.088

Length of hospi- $\quad 4.90 \pm 1.43$

$4.96 \pm 1.38$

0.553

tal(days)

Score of PHQ-9 $\quad 1.46 \pm 2.09$

$2.02 \pm 2.22$

$0.000^{*}$

Table 2Adverse Complication 


$$
\begin{array}{llll}
2020 & 2020 & 2019 & 2019 \\
(n=347) & (n=347) & (n=475) & (n=475)
\end{array}
$$
n
(\%)
$\mathbf{n}$

(\%)

Number of 152

43.80

191

40.21

0.302

caesarean

Macrosomia 42

12.10

12

2.53

0.000*

Gestational 63 diabetes mellitus

Gestational 27 hypertension

Fetal dis- 29 tress
8.36

18.16

108

22.74

0.110

7.78

35

7.37

0.825

28

5.89

0.170 


$$
\begin{array}{llll}
2020 & 2020 & 2019 & 2019 \\
(n=347) & (n=347) & (n=475) & (n=475)
\end{array}
$$

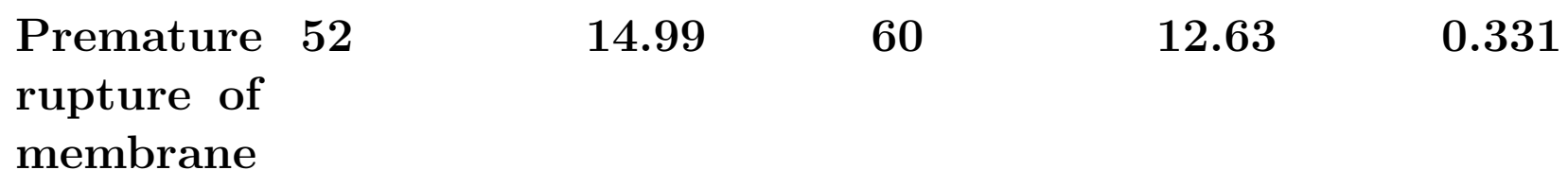

$\begin{array}{llllll}\text { Premature } & 20 & 5.76 & 24 & 5.05 & 0.655\end{array}$
labour

Precipitate 22

6.34

40

8.42

0.588

labour

Postpartum 19

5.48

25

5.26

0.894

hemorrhage

Table 3 Neonatal birth weight 


$$
2020(n=355) 2019(n=486) \quad \mathrm{P}
$$

Birth weight (g) $\quad 3440.96 \pm 540.22 \quad 3371.55 \pm 526.24 \quad 0.062$ 